\title{
Evaluation of a Thermal-Based Flow Meter for Assessment of Mobile Resting Metabolic Rate Measures
}

\author{
Nai-Yuan Liu $\mathbb{D}^{1,2}$ Yue Deng, ${ }^{1,2}$ Francis Tsow, ${ }^{2}$ Devon Bridgeman, ${ }^{2}$ Xiaojun Xian, ${ }^{2}$ \\ Jane J. Dean, ${ }^{3}$ Janet L. Wilson, ${ }^{3}$ Nongjian Tao, ${ }^{2}$ Doina Kulick ${ }^{(D},{ }^{3}$ and Erica Forzani ${ }^{1,2}$ \\ ${ }^{1}$ School for Engineering of Matter, Transport and Energy, Arizona State University, Tempe, AZ, USA 85287 \\ ${ }^{2}$ Center for Bioelectronics and Biosensors, Biodesign Institute, Arizona State University, Tempe, AZ, USA 85287 \\ ${ }^{3}$ Mayo Clinic Hospital, Scottsdale, AZ, USA 85287
}

Correspondence should be addressed to Doina Kulick; kulick.doina@mayo.edu and Erica Forzani; eforzani@asu.edu

Received 8 April 2018; Revised 10 July 2018; Accepted 14 August 2018; Published 9 October 2018

Academic Editor: Eduard Llobet

Copyright ( $) 2018$ Nai-Yuan Liu et al. This is an open access article distributed under the Creative Commons Attribution License, which permits unrestricted use, distribution, and reproduction in any medium, provided the original work is properly cited.

\begin{abstract}
This work evaluates the use of a new flow meter to assess exhalation rate. A mobile indirect calorimeter (MIC) was designed and used to measure resting metabolic rate (RMR), which relies on the measure of $\mathrm{O}_{2}$ consumption rate $\left(\mathrm{VO}_{2}\right)$ and $\mathrm{CO}_{2}$ production rate $\left(\mathrm{VCO}_{2}\right)$. The device was produced from a commercially available and well-established indirect calorimeter and implemented with a new flow meter for the purpose of this study. $\mathrm{VO}_{2}$ and $\mathrm{VCO}_{2}$ were assessed by measuring exhalation rates using the new flow meter and $\mathrm{O}_{2}$ and $\mathrm{CO}_{2}$ concentrations in breath using the original colorimetric sensors of the indirect calorimeter. The new flow meter was based on a thermal flow meter (TFM) affixed to an orifice with a diameter of $6.8 \mathrm{~mm}$ used as a passage for exhaled breath from 16 subjects. The results were compared with a metabolic cart (Medical Graphics), which was connected in series to the modified device. We found that $69 \%$ of the results had more than a $10 \%$ difference between the modified MIC device and the reference instrument, suggesting that the sensitivity of the thermal flow meter changed over time, which precluded its use as a flow meter for breath flow rate measurement.
\end{abstract}

\section{Introduction}

Resting metabolic rate (RMR), also known as resting energy expenditure (REE), is defined as the amount of energy expended by a person at rest [1]. RMR typically represents over $65 \%$ of total daily energy expenditure (TEE), and the percentage can be as high as $80-90 \%$ of TEE for sedentary people [2]. Therefore, evaluating an individual's RMR is important to help assess the daily caloric intake need for weight management. The most well-established method to assess RMR is indirect calorimetry, which determines RMR based on the oxygen consumption rate $\left(\mathrm{VO}_{2}\right)$ and carbon dioxide production rate $\left(\mathrm{VCO}_{2}\right)$, via the Weir equation [3]. Traditional indirect calorimetry instruments, such as metabolic carts, are expensive and bulky and require frequent calibration and trained personnel for correct use. To avoid using such equipment, equations were created to estimate RMR from a person's age, gender, weight, and height [4]. However, it has been shown that the use of these equations could result in estimations that are $600-900 \mathrm{kcal} /$ day off from the true values measured with indirect calorimetry [5].

To overcome the above problem, a mobile indirect calorimeter was developed to facilitate personal use for RMR tracking $[6,7]$. The device uses a differential-pressure-based flow meter and a colorimetric-based chemical sensor to determine $\mathrm{VO}_{2}$ and $\mathrm{VCO}_{2}$. Breath is delivered into the sensing chamber, where the $\mathrm{O}_{2}$ and $\mathrm{CO}_{2}$ react with a sensor chip, inducing color changes for determining exhalation $\mathrm{O}_{2}$ and $\mathrm{CO}_{2}$ concentrations. $\mathrm{VO}_{2}$ and $\mathrm{VCO}_{2}$ were calculated based on the breath $\mathrm{O}_{2}$ and $\mathrm{CO}_{2}$ concentrations and breath flow rate. The performance of the device and colorimetric sensor has been validated by over 300 measurements against the gold standard, the Douglas bag method [7]. In addition, this mobile indirect calorimeter has been further used in human subjects to confirm functionality $[8,9]$.

In order to investigate an alternative to the existing technology, we modified the commercial device to explore the utility of a new flow sensor for breath analysis. 
Some commonly used flow meters are described as follows: (1) Fleisch-type meters use small capillary tubes to create laminar flow, which provide good linear relation, but they suffer from clogging and are hard to clean [10]. (2) Orifice-differential-pressure-sensor-based flow meters are simple, robust, and inexpensive, but they might offer high flow resistance [11]. (3) Variable-orifice flow meters provide less flow resistance by having an orifice size proportional to the flow [12]. However, they are prone to wearing out mechanically over time. (4) Pitot tube flow meters are inexpensive and have very low flow resistance, as their flow rate is proportional to the stagnation pressure. However, the sensitivity of this kind of tube is usually very low and is limited to low and steady flows [13]. While the field of flow meters has been well studied, flow measurement for breathing conditions with a wide range of flow rates has not been reported systematically, and it is important to investigate the direct breath flow measurement due to special conditions such as temperatures above room temperature and possible condensation from humidity.

In this work, we used an orifice plate (diameter of $6.8 \mathrm{~m}$ ) combined with a thermal flow meter with the inlet and outlet located before and after the orifice plate for breath flow rate measurement. The thermal flow meter converted differential thermal energy change into flow rate [14]. Unlike other flow meters, a thermal flow meter enables to work at low flow resistances. A comparative study between this modified device and a metabolic cart was conducted to validate the performance of the new system.

\section{Methods}

2.1. The MIC from This Work. The MIC measured $\mathrm{VO}_{2}$ and $\mathrm{VCO}_{2}$ from breath and determined RMR according to the Weir equation [15]. When breath $\mathrm{O}_{2}$ and $\mathrm{CO}_{2}$ flow through the sensor chip, they induced a color change on the sensor because of specific chemical reactions. The device had a light source and photodiodes to determine the absorbance change during the chemical reaction. The photodiodes transduced the absorbance of the sensor to a digital signal, which was wirelessly transmitted to a smart device for data processing. The absorbance was calculated by taking the negative logarithm of the signal response from the sensing area $\left(S_{\text {sens. }}\right)$ divided by the signal response from the reference area $\left(S_{\text {ref. }}\right)$ as follows:

$$
\text { Absorbance }=-\log \left(\frac{S_{\text {sens. }}}{S_{\text {ref. }}}\right) .
$$

A built-in calibration curve converted the absorbance to corresponding fraction of exhaled $\mathrm{O}_{2}$ concentration $\left(\mathrm{FEO}_{2}\right)$ and fraction of exhaled $\mathrm{CO}_{2}$ concentration $\left(\mathrm{FECO}_{2}\right)$. As mentioned above, our new flow meter implemented into the device allowed the measurement of the exhalation breath rate (VE), which was used to calculate the oxygen consumption rate $\left(\mathrm{VO}_{2}\right)$, carbon dioxide production rate $\left(\mathrm{VCO}_{2}\right)$, and RMR through the following equations:

$$
\begin{aligned}
\mathrm{VO}_{2}= & \left(0.2093-\mathrm{FEO}_{2}\right) \cdot \mathrm{VE}, \\
\mathrm{VCO}_{2}= & \left(\mathrm{FECO}_{2}-0.0003\right) \cdot \mathrm{VE}, \\
\operatorname{RMR}(\mathrm{kcal} / \text { day })= & {\left[3.941\left(\mathrm{VO}_{2}(\mathrm{~L} / \mathrm{min})\right)\right.} \\
& \left.+1.11\left(\mathrm{VCO}_{2}(\mathrm{~L} / \mathrm{min})\right)\right] * 1.440,
\end{aligned}
$$

where $\mathrm{FEO}_{2}$ is the breath $\mathrm{O}_{2}$ concentration typically measured between 0.13 and 0.19 and $\mathrm{FECO}_{2}$ is the breath $\mathrm{CO}_{2}$ concentration typically measured between 0.03 and 0.06 . The fraction concentration of $\mathrm{O}_{2}$ in the atmosphere is assumed to be 0.2093 , and that of $\mathrm{CO}_{2}$ to be 0.0003 based on the typical atmospheric condition [16]. Since the inhalation volume and exhalation volume are very close, we also assumed that they were the same in the calculation. Lastly, a built-in fan dried the flow tube to avoid water condensation buildup between measurements.

2.2. The Flow Meter on This Work's MIC. The flow meter of the MIC, TFM, was an off-the-shelf electronic component obtained from Omron ${ }^{\circledR}$ (part number: D6F-P0010A2 [17]). It was located with the inlet and outlet connected to the exhaled breath channel in the upstream and downstream portion at each side of the $6.8 \mathrm{~mm}$ orifice plate. The temperature output from a thermopile or thermistor in the TFM is based on its electrical voltage change, which was subsequently measured by the microcontroller analog-to-digital converter. The TFM utilized the transduction of energy change to voltage change to measure flow rate. Usually in a TFM, there are two thermistors in the flow tube: the reference thermistor, which measures the temperature of the incoming gas called the reference temperature $\left(T_{0}\right)$, and the hot thermistor, which is driven by a current to raise its temperature $\left(T_{\mathrm{R}}\right)$ to some fixed level above the temperature of the gas [14]. In still gas, the hot thermistor loses heat due to energy dissipation (both radiation and convection) to balance the electrical energy that heats the thermistor, stabilizing the hot thermistor's temperature. When the gas is flowing, the heat dissipation constant $(K)$ of the hot thermistor increases and $T_{R}$ decreases, resulting in an increase in the flow of current. The driving circuit senses the change in the current and raises the voltage $(V)$ across the hot thermistor to maintain a fixed temperature difference between the reference thermistor and the hot thermistor, which is described by the following equation $[18,19]$.

$$
V=\sqrt{K R\left[T_{\mathrm{R}}-T_{0}\right]},
$$

where $R$ is the resistance.

Alternatively, two thermopiles (one upstream and the other downstream of the gas flow) and a heating element in between the thermopiles can be used. This approach can simplify the required circuitry. When there is no gas flow, the temperature as measured by the two thermopiles is the same. As gas flows, the upstream temperature $\left(T_{0}\right)$ is expected to be lower than the downstream temperature $\left(T_{R}\right)$. The resulting temperature profile converted to voltages will allow gas flow 


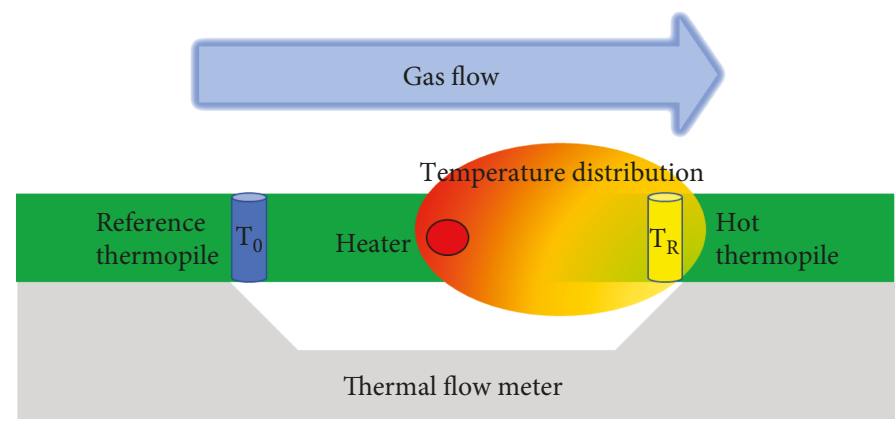

Figure 1: Schematic of a thermal flow meter.

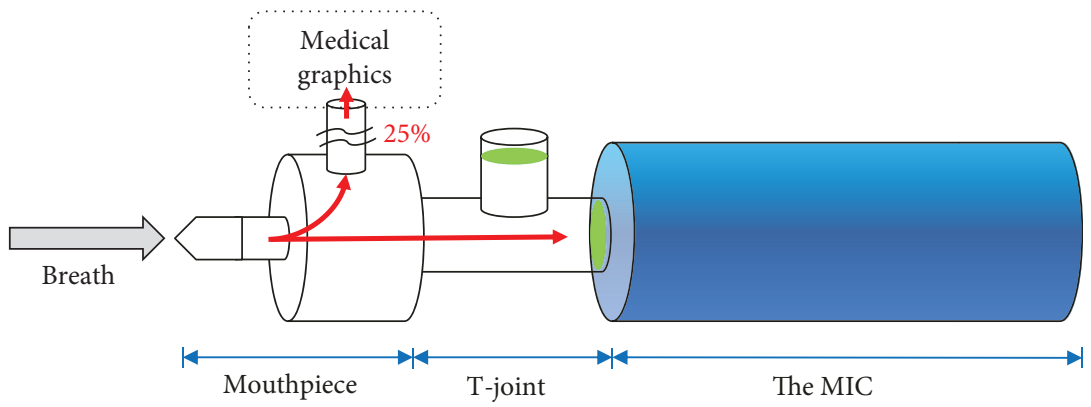

FIgure 2: The schematic of sequence of how the MIC is connected to the MG.

rate to be determined. The TFM used in this project uses the thermopile approach (Figure 1).

After placing the new TMF in the MIC, a calibration curve was built between the voltage and flow rate for a range from $20 \mathrm{~L} / \mathrm{min}$ to $120 \mathrm{~L} / \mathrm{min}$, using dry air. The flow rate was integrated over time to assess the total volume which was used as the criteria to determine the amount of breath to be used. The measurement of flow was set to be stopped when a total volume of $30 \mathrm{~L}$ was collected. The reference flow used in the calibration was a commercial flow sensor (Mass Flow Sensor SFM3000, Sensirion).

2.3. Metabolic Cart Used as a Reference Instrument. Metabolic carts are commonly used in hospitals to measure the individual's RMR. Here, we used Medical Graphics (MG) Ultima $^{\mathrm{TM}} \mathrm{Cardi}^{\circledR}$ as the reference equipment. This instrument provides RMR measurement based on breath-by-breath analysis, is FDA-cleared, and is typically used for assessments of patients' RMR or energy expenditure under exercise conditions at institutions such as Mayo Clinic. To validate the new MIC, 16 subjects were recruited (see details below) and measured by connecting the mouthpiece of the MG to the MIC in sequence with a T-joint, which only allowed exhalation breath to go through (Figure 2). Unlike the MIC, the MG only samples in an average of $\sim 25 \%$ of the breath for breath $\mathrm{O}_{2}$ and $\mathrm{CO}_{2}$ measurement. Therefore, a factor of 1.25 was applied to the exhalation rate (VE) measured by the MIC (accordingly to $\mathrm{VO}_{2}, \mathrm{VCO}_{2}$, and RMR) to compensate for the loss of the breath.

2.4. Subjects. Sixteen (16) healthy adults, including 9 males and 6 females, were included in the study. The number of subjects was chosen to discriminate average RMR of 1800 and $2000 \mathrm{kcal} /$ day with a standard deviation of $200 \mathrm{kcal} /$ day (typical clinical variability) and to reach a power of 0.80 and an alpha of 0.05. The subjects' age ranged from 27 to 57 years, and BMIs ranged from 18 to $46 \mathrm{~kg} / \mathrm{m}^{2}$. The measurements of the resting metabolic rate were performed early in the morning following a standard clinical protocol approved by the Institutional Review Board of Mayo Clinic (IRB protocol number 16-003321). The protocol required subjects to fast with no strenuous exercise for the last 12 hours or no moderate exercise for 4 hours prior to the measurement. The subjects remained in a comfortable sitting position in a darkened room with room temperature at $\sim 23^{\circ} \mathrm{C}$. The subjects were asked to rest for 30 minutes before the measurement and then to breathe normally through the setup (Figure 2) and during the measurement.

\section{Results}

Based on the experience from other studies [5, 8, 9], predicative RMR equations such as Mifflin-St. Jeor or HarrisBenedict are average population estimations based on the physical characteristics of the subjects (age, gender, height, and weight) and do not necessarily represent actual measured RMR values. In the case of this study, similar conclusions have been found. Table 1 shows the comparison between the RMR calculated by the Mifflin-St. Jeor equation (MSJE) [20] and the RMR measured with our reference instrument, Medical Graphics (MG). As it can be observed, absolute differences ranging from $20 \%$ to $41 \%$ were observed for most of the subjects $(11 / 16, \sim 68 \%$ of the subjects). These 
TABLE 1

\begin{tabular}{|c|c|c|c|c|c|c|c|c|}
\hline $\begin{array}{l}\text { Subject } \\
\text { number }\end{array}$ & $\begin{array}{l}\text { Weight } \\
(\mathrm{kg})\end{array}$ & $\begin{array}{l}\text { Height } \\
(\mathrm{cm})\end{array}$ & $\begin{array}{l}\text { Age } \\
(\mathrm{yr})\end{array}$ & Gender & $\begin{array}{l}\text { MSJE RMR } \\
\text { (kcal/day) }\end{array}$ & $\begin{array}{l}\text { MG RMR } \\
\text { (kcal/day) }\end{array}$ & $\begin{array}{l}\text { Difference: MG RMR - } \\
\text { MSJE RMR (kcal/day) }\end{array}$ & $\begin{array}{c}\text { Differential percentage: } \\
\text { (MG RMR - MSJE RMR) } \\
\text { /MG RMR *100\% }(\%)\end{array}$ \\
\hline 1 & 68 & 170 & 46 & $\mathrm{~F}$ & 1968 & 1758 & -210 & -11 \\
\hline 2 & 85 & 190 & 27 & $\mathrm{M}$ & 2335 & 2547 & 211 & 9 \\
\hline 3 & 60 & 163 & 27 & $\mathrm{~F}$ & 1751 & 1997 & 246 & 14 \\
\hline 4 & 84 & 189 & 27 & M & 2319 & 2983 & 664 & 29 \\
\hline 5 & 61 & 164 & 27 & $\mathrm{~F}$ & 1767 & 2315 & 548 & 31 \\
\hline 6 & 66 & 161 & 59 & $\mathrm{~F}$ & 1956 & 1150 & -806 & -41 \\
\hline 7 & 111 & 180 & 47 & M & 2631 & 2133 & -498 & -19 \\
\hline 8 & 48 & 161 & 50 & $\mathrm{~F}$ & 1732 & 1366 & -366 & -21 \\
\hline 9 & 83 & 176 & 58 & M & 2381 & 1694 & -687 & -29 \\
\hline 10 & 150 & 183 & 57 & M & 3089 & 2261 & -827 & -27 \\
\hline 11 & 66 & 161 & 54 & $\mathrm{~F}$ & 1931 & 1218 & -713 & -37 \\
\hline 12 & 102 & 182 & 52 & M & 2578 & 1700 & -879 & -34 \\
\hline 13 & 102 & 159 & 45 & $\mathrm{~F}$ & 2234 & 1706 & -528 & -24 \\
\hline 14 & 101 & 175 & 56 & M & 2544 & 2510 & -35 & -1 \\
\hline 15 & 78 & 186 & 57 & M & 2388 & 1808 & -580 & -24 \\
\hline 16 & 110 & 186 & 57 & M & 2708 & 1983 & -725 & -27 \\
\hline
\end{tabular}

MSJE RMR = resting metabolic rate calculated based on the Mifflin-St. Jeor equation: for women: $10 \times$ weight $(\mathrm{kg})+6.25 \times$ height $(\mathrm{cm})-5 \times$ age $(\mathrm{y})-161$; for men: $10 \times$ weight $(\mathrm{kg})+6.25 \times$ height $(\mathrm{cm})-5 \times$ age $(\mathrm{y})+5 . \mathrm{MG}$ RMR $=$ resting metabolic rate measured by Medical Graphics.

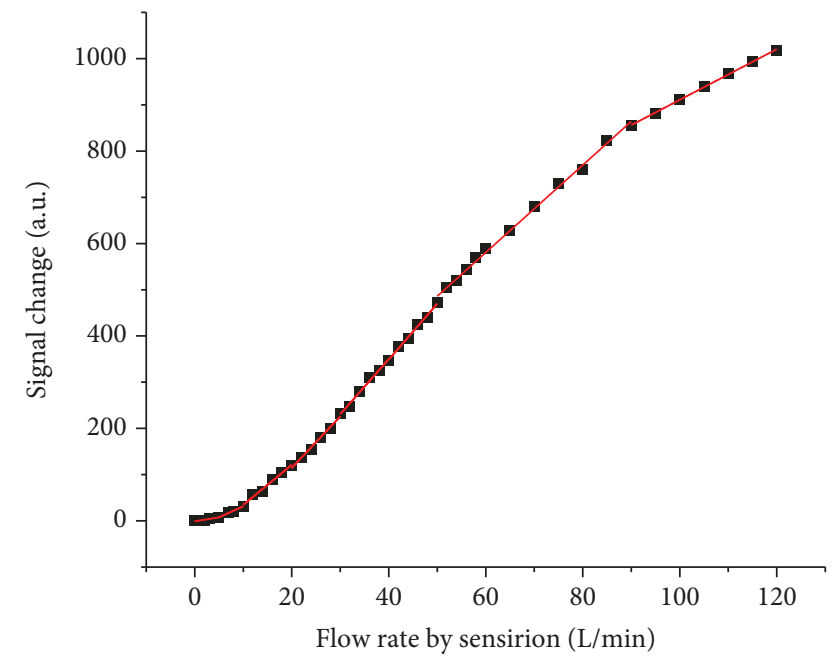

FIgURE 3: The calibration curve of the flow rate ranging from 0 to $120 \mathrm{~L} / \mathrm{min}$.

differences translated into energy expenditure assessments with differences between 300 and $880 \mathrm{kcal} /$ day, which is a significant amount that could cause weight gain in someone targeting weight loss. For this reason, we believe that the only way to assess the true RMR of an individual is through measuring it. Indirect calorimetry is the recommended method. Therefore, the goal of this paper is to compare the results between a modified self-designed device and a solid wellestablished reference indirect calorimetry method.

Figure 3 shows the calibration curve of the new MIC. Since the built-in calibration curve ranging from 0 to $120 \mathrm{~L} / \mathrm{min}$ was not linear, it was divided into 7 linear

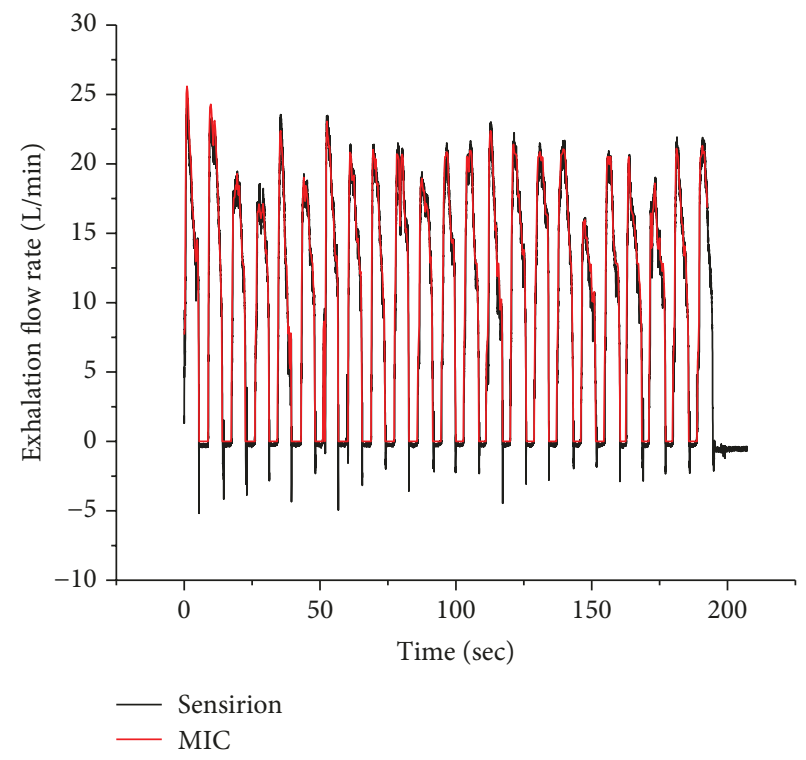

FIgURE 4: A breath-by-breath exhalation flow rate monitoring was measured by our modified device and Sensirion.

segments $(0-10,10-20,20-30,30-40,40-50,50-90$, and $90-120 \mathrm{~L} / \mathrm{min})$ to optimize the performance of the firmware of the device and reduce calculation time.

Figure 4 shows the example of breath exhalation flow rate (VE) measured by the MIC and the reference Sensirion flow sensor. The exhalation flow rate measured by the new MIC was recovered by using the calibration curve built from the Sensirion flow sensor and corrected by standard pressure and dry conditions before the comparison. The interrogation 


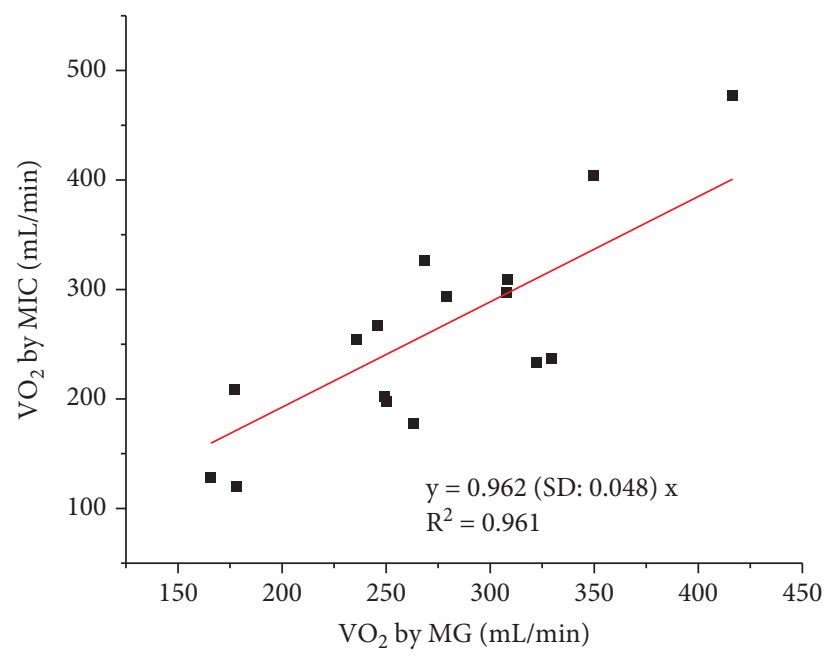

(a)

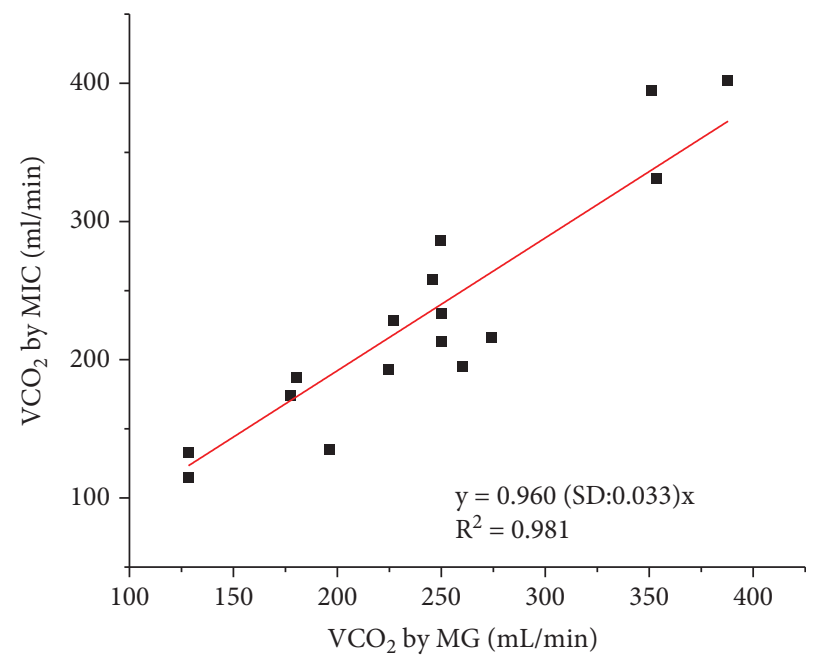

(c)

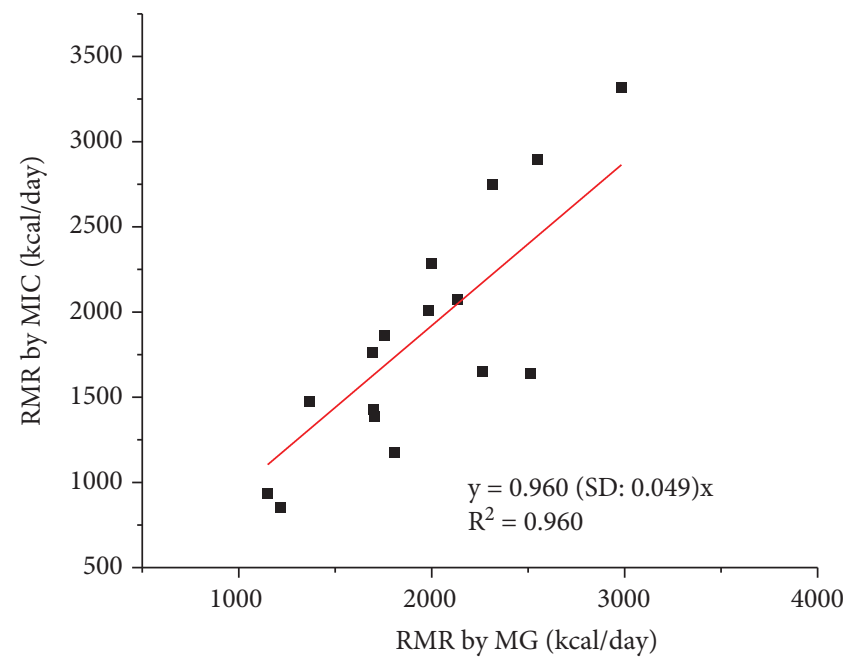

(e)

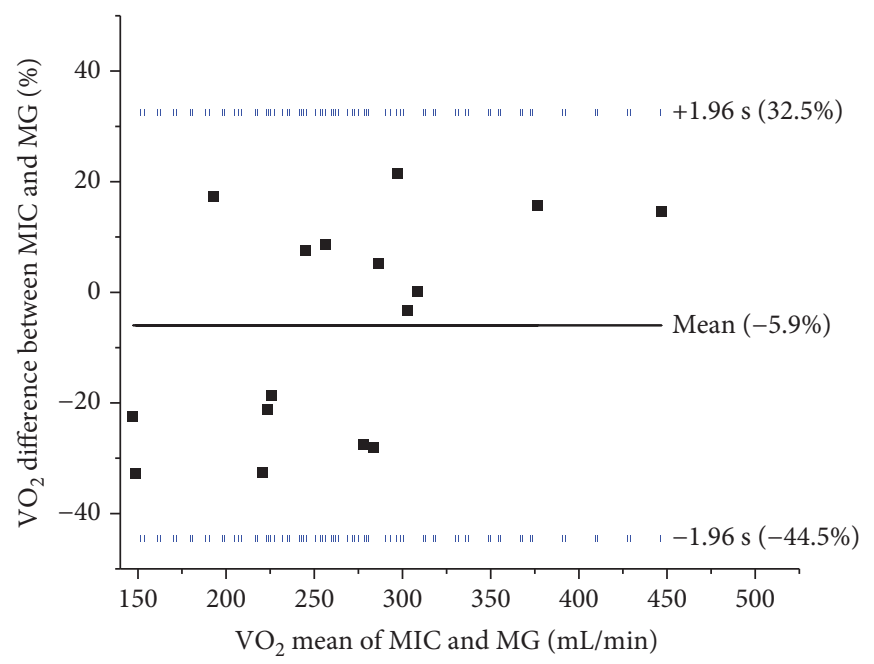

(b)

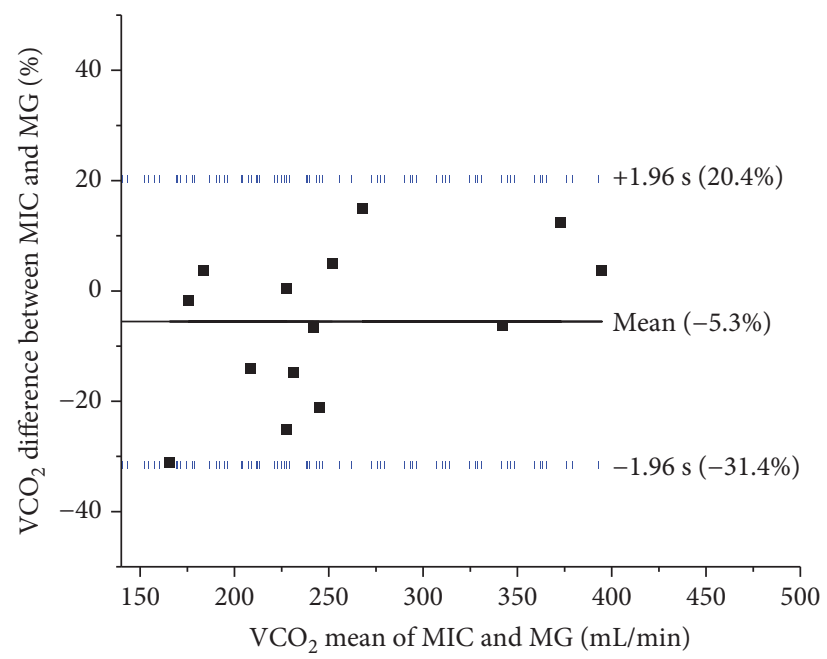

(d)

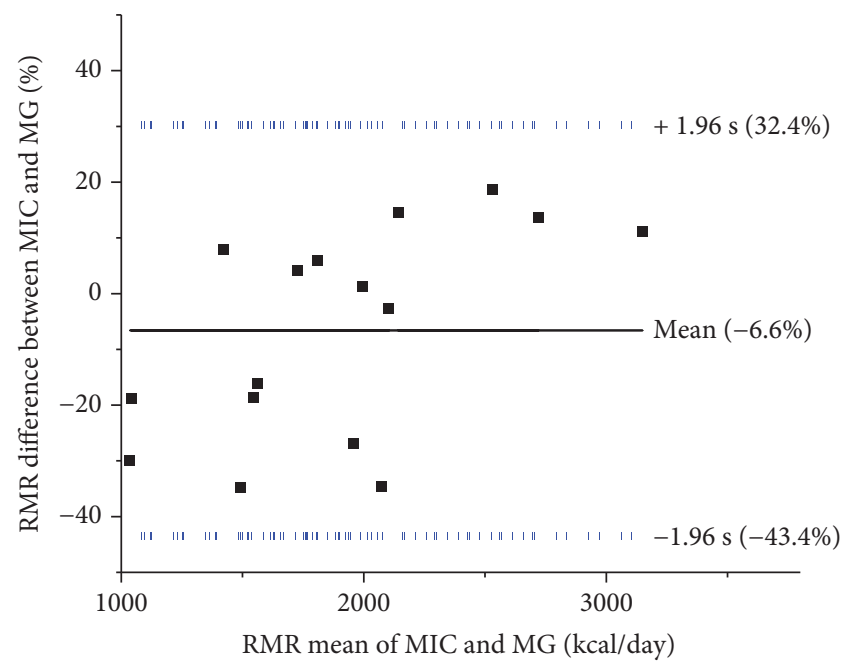

(f)

FIGURE 5: Comparison between the new MIC and the MG instrument. Linear correlations and Bland-Altman analysis for $\mathrm{VO}_{2}(\mathrm{a}, \mathrm{b})$, $\mathrm{VO}_{2}$ (c, d), and RMR (e, f). 


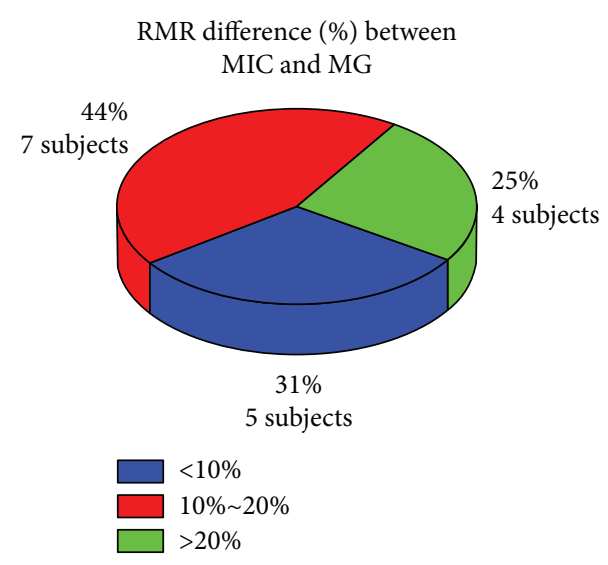

FIGURE 6: Pie chart distribution for RMR difference (\%) between the modified MIC and MG.

showed the ability of the device to monitor exhalation flow rate in a breath-by-breath manner. Note that this accuracy was maintained during the first 10 weeks of the use of the flow meter. However, a degradation of performance was observed over time as shown as follows.

To evaluate the performance of the new MIC over time, 16 subjects' RMR were measured using the MIC and the MG simultaneously. As mentioned before, a factor of 1.25 was applied. Figures 5(a), 5(c), and 5(d) show that $\mathrm{VO}_{2}$, $\mathrm{VCO}_{2}$, and RMR measured by the new MIC and the MG correlated very well. All of them had a regression coefficient $\left(R^{2}\right)$ greater than 0.96 . On average, the measured RMR from the MIC were about 5.9\% less than that from the MG for $\mathrm{VO}_{2}$, $5.3 \%$ for $\mathrm{VCO}_{2}$, and $6.6 \%$ for RMR. Figures 5(b), 5(d), and 5 (f) show the Bland-Altman plot of the percentage difference between the new MIC and the MG for $\mathrm{VO}_{2}, \mathrm{VCO}_{2}$, and RMR defined as (MIC $-\mathrm{MG}) / \mathrm{MG} * 100 \%$. All the results for $\mathrm{VO}_{2}$, $\mathrm{VCO}_{2}$, and $\mathrm{RMR}$ were within the range of the mean value \pm 1.96 standard deviation, showing a reasonable performance. However, we identified that $\pm 10 \%$ should be a practical acceptance limit of performance since $\pm 10 \%$ is the physiological variability of RMR [16], and therefore, percentage differences larger than $\pm 10 \%$, which were observed for $\mathrm{VO}_{2}, \mathrm{VCO}_{2}$, and RMR, were found to be inadequate for the acceptance criteria of good performance.

In order to quantify the lack of the new MIC device's performance, we further analyzed the results, defining three categories based on absolute percentage differences: (a) $<10 \%$, (b) $10-20 \%$, and (c) $>20 \%$. Figure 6 shows a pie chart that categorizes the absolute percentage difference between the measured results from the new MIC and the MG. Twenty-five percent (25\%) of the measurements had a difference larger than 20\%,44\% had a difference between 10 and $20 \%$, and only $31 \%$ had a difference within $\pm 10 \%$. In summary, $69 \%$ of the measurements had a difference of over $10 \%$.

As mentioned before, we analyzed the exhaled $\mathrm{O}_{2}$ and $\mathrm{CO}_{2}$ concentration outputs from the modified MIC vs. the $\mathrm{MG}$, and no significant difference was found (not shown). However, we found the difference to be caused by VE (see more details below). In fact, we noticed a degradation of the VE performance from the TFM over time. Figure 7 shows

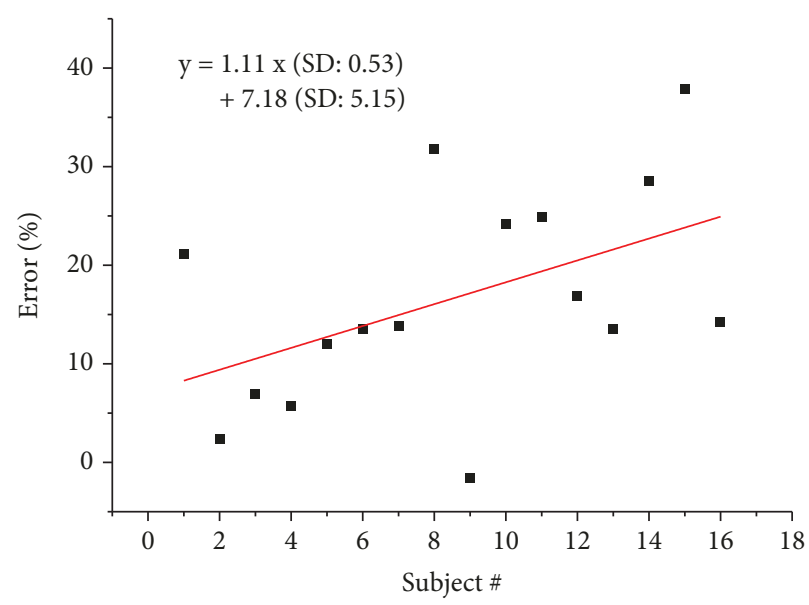

FIgURE 7: Absolute percentage difference of VE between the MG and MIC calculated as error $(\%)=\left|\left(\mathrm{VE}_{\mathrm{MIC}}-\mathrm{VE}_{\mathrm{MG}}\right) / \mathrm{VE}_{\mathrm{MG}} * 100 \%\right|$ for each subject. The subjects $1-16$ were measured between a period in time starting on February 20, 2017, and ending on February 05, 2018.

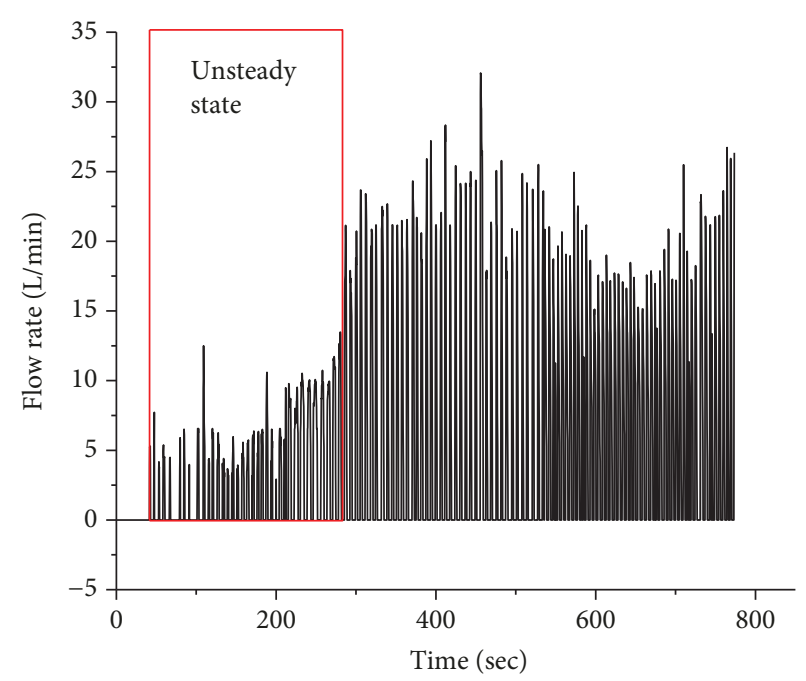

FIGURE 8: An example of a breath flow rate recording on the modified MIC after the performance degradation was detected.

the error of VE between MG and MIC on each subject over the period of use. A clear increase in error was built up over time (as the number of subjects increased), with VE measured from the modified MIC systematically lower with respect to $\mathrm{VE}$ from $\mathrm{MG}$.

Figure 8 shows a breath-by-breath example of the measurement of a subject with the modified MIC after the performance degradation was detected. As opposite to the patterns observed before the degradation where relatively constant maximum exhalation rates were observed in each cycle from the beginning to the end of the measurement (see Figure 4), this pattern shows significantly lower exhalation rates at the beginning of the measurement. The recorded flow rate before 280 seconds was about 2 times lower than the flow rate recorded after in the remaining second portion of the measurement. Further investigation needs to be done to learn 
more about this phenomenon, and only a hypothesis can be discussed at this time.

A possible reason for failure is that the condensation of moisture in breath over time may cause damage in the TFM's thermopile and the mechanisms involved to reach adequate behavior. Before the start of the measurement, the humidity around the thermopiles is the same as the humidity of the environment. After the measurement starts, a sudden disturbance from the moisture of breath condensing on the surface of thermopiles takes place.

In a new TFM, the thermopile seems to be immune to humidity condensation (probably due to hydrophobic coatings). In a used TFM, the thermopiles may suffer from a damage of their coatings and a breath humidity condensation, which is a phase change that releases heat, and may dramatically change the energy flow, changing the TFM working conditions and causing an erroneous reading. As the measurement continues, the condensation reaches a steady state and no longer perturbates the system with further heat release, and therefore, the temperature in the system would reach equilibrium so that the TFM can function correctly.

Using tubing before the flow meter inlet to trap humidity could possibly reduce the condensation onto the thermopiles. In addition, a systematical correction algorithm can also be applied to accommodate the effect of condensation. These modifications are possibilities to mitigate the abovementioned problems. However, the TFM in its current form is not suitable to be used as a flow meter for a gas sample that has high humidity or temperatures significantly different from ambient temperatures.

\section{Conclusion}

To conclude, we observed a $6.6 \%$ underestimation of the RMR output in the new MIC. Although the initial calibration of the TFM sensor done at a range from 0 to $120 \mathrm{~L} / \mathrm{min}$ rendered accurate values with respect to reference commercial flow meters, the original calibration failed to be applied after using the TFM over a period of 3-4 months. Furthermore, $\mathrm{VO}_{2}$ and $\mathrm{VCO}_{2}$ were measured to render RMR measures by the modified MIC and MG in parallel, and $69 \%$ of the measurements showed a difference between the two methods that was greater than $10 \%$, which could have been caused by moisture from the breath collection or from the degradation of the TFM sensitivity to the flow. Further investigation needs to be done to confirm this assumption. Nevertheless, the TFM is not suitable for highly humid gas sample measurement, such as human breath.

\section{Data Availability}

The data used to support the findings of this study are included within the article.

\section{Conflicts of Interest}

E.F., X.J., D.B., F.T., and N.T. work for Breezing ${ }^{\mathrm{TM}}$, an indirect calorimeter tracker used as a base device of the modified indirect calorimeter device studied in this work. All other authors declare that they have no conflict of interest.

\section{Authors' Contributions}

Nai-Yuan Liu and Yue Deng contributed equally to this work.

\section{Acknowledgments}

The authors would like to thank Arizona State University and Mayo Clinic for their support.

\section{References}

[1] A. C. Pinheiro Volp, F. C. Esteves de Oliveira, R. Duarte Moreira Alves, E. A. Esteves, and J. Bressan, "Energy expenditure: components and evaluation methods," Nutricion Hospitalaria, vol. 26, pp. 430-440, 2011.

[2] E. Ferranini, "The theoretical bases of indirect calorimetry: a review," Metabolism Clinical and Experimental, vol. 37, no. 3, pp. 287-301, 1988.

[3] R. D. Gupta, R. Ramachandran, P. Venkatesan, S. Anoop, M. Joseph, and N. Thomas, "Indirect calorimetry: from bench to bedside," Indian Journal of Endocrinology and Metabolism, vol. 21, no. 4, pp. 594-599, 2017.

[4] J. A. Harris and F. G. Benedict, "A biometric study of human basal metabolism," Proceedings of the National Academy of Sciences of the United States of America, vol. 4, no. 12, pp. 370-373, 1918.

[5] Y. Deng, B. Scott, and S. T. S. Jeor, "Comparison of resting metabolic rates between predictive euqations and portable indirect calorimeter," in Obesity Week, Washington, DC, USA, 2017, https://obesityweek.com/about/.

[6] D. Zhao, X. Xian, M. Terrera et al., "A pocket-sized metabolic analyzer for assessment of resting energy expenditure," Clinical Nutrition, vol. 33, no. 2, pp. 341-347, 2014.

[7] X. Xian, A. Quach, D. Bridgeman, F. Tsow, E. Forzani, and N. Tao, "Personalized indirect calorimeter for energy expenditure (EE) measurement," Global Journal of Obesity, Diabetes and Metabolic Syndrome, vol. 2, pp. 4-8, 2014.

[8] D. Jackemeyer, E. Forzani, and C. Whisner, "Study of resting energy expenditure and weight changes during pregnancy," Global Journal of Obesity, Diabetes and Metabolic Syndrome, vol. 4, pp. 16-23, 2017.

[9] C. S. Stump, D. Jackemeyer, Y. Abidov, K. Herbst, N. Tao, and E. Forzani, "Study of the effect of mobile indirect calorimeter on weight management," Global Journal of Obesity, Diabetes and Metabolic Syndrome, vol. 4, pp. 44-50, 2017.

[10] J. W. Kreit and F. C. Sciurba, "The accuracy of pneumotachograph measurements during mechanical ventilation," American Journal of Respiratory and Critical Care Medicine, vol. 154, no. 4, pp. 913-917, 1996.

[11] B. Raton and R. Goldstein, Fluid Mechanics Measurements, CRC Press, Boca Raton, FL, USA, 1996.

[12] J. S. Gravenstein, M. B. Jaffe, N. Gravenstein, and D. A. Paulus, Capnography, Cambridge University Press, Cambridge, England, 2011.

[13] P. J. LaNasa and E. L. Upp, Fluid Flow Measurement: A Practical Guide to Accurate Flow Measurement, Butterworth-Heinemann, Oxford, England, 2014. 
[14] B. Smith, "Gas-metering alternatives emerge for the connected world," Electronic Design, 2010, https://www.electronicdesign. $\mathrm{com} /$.

[15] J. B. D. Weir, "New methods for calculating metabolic rate with special reference to protein metabolism," The Journal of Physiology, vol. 109, no. 1-2, pp. 1-9, 1949.

[16] W. D. McArdle, F. I. Katch, and V. L. Katch, Exercise Physiology: Nutrition, Energy, and Human Performance, Lippincott Williams \& Wilkins, 2010.

[17] L. Thomas, M. Lind, and D. Kulick, "Assessment survey of high impact biomarkers for real-time monitoring," Internal Review at Mayo Clinic, Scottsdale, AZ, USA, 2015.

[18] A. J. Skinner and M. F. Lambert, "Evaluation of a warmthermistor flow sensor for use in automatic seepage meters," IEEE Sensors Journal, vol. 9, no. 9, pp. 1058-1067, 2009.

[19] A. C. M. C. Admésio, M. Ricardo, and A. Q. Divo, "Thermistor based, low velocity isothermal, air flow sensor," Measurement Science and Technology, vol. 27, no. 3, article 035307, 2016.

[20] M. D. Mifflin, S. T. St Jeor, L. A. Hill, B. J. Scott, S. A. Daugherty, and Y.O. Koh, "A new predictive equation for resting energy expenditure in healthy individuals," The American Journal of Clinical Nutrition, vol. 51, no. 2, pp. 241-247, 1990. 


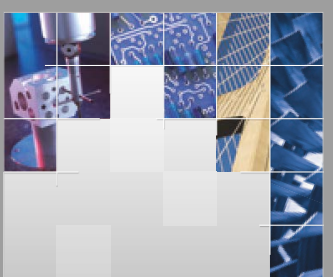

\section{Enfincering}
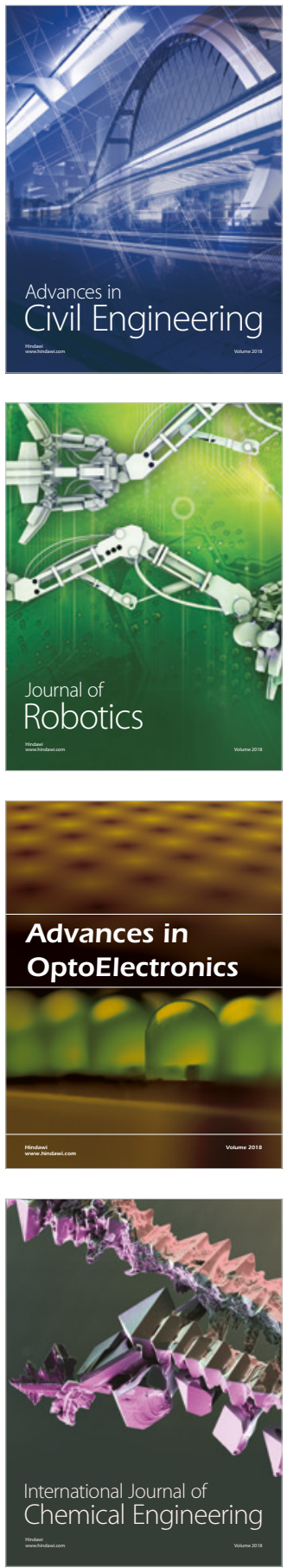

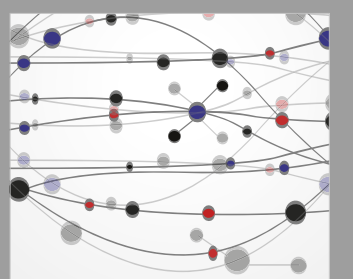

\section{Rotating \\ Machinery}

The Scientific World Journal

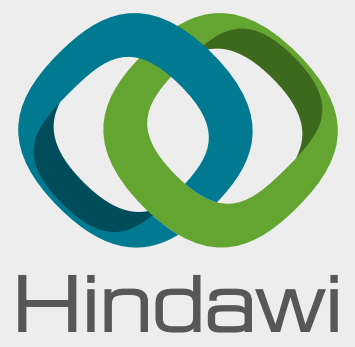

Submit your manuscripts at

www.hindawi.com
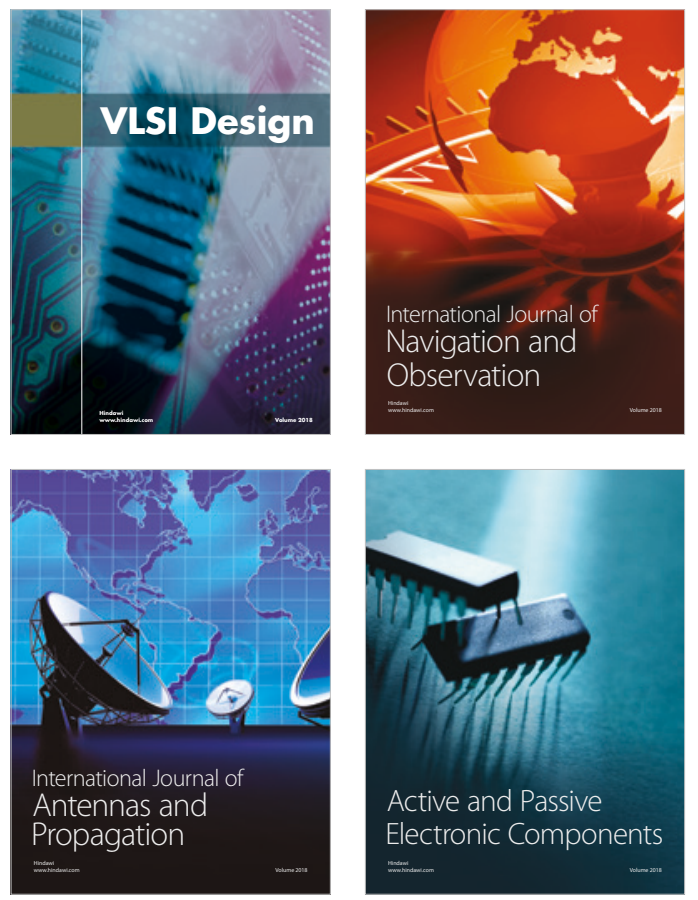
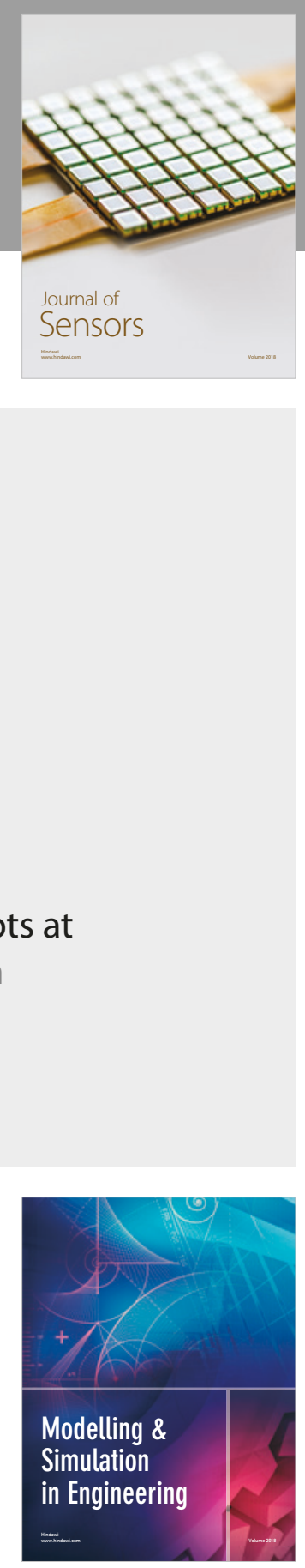

\section{Advances \\ Multimedia}
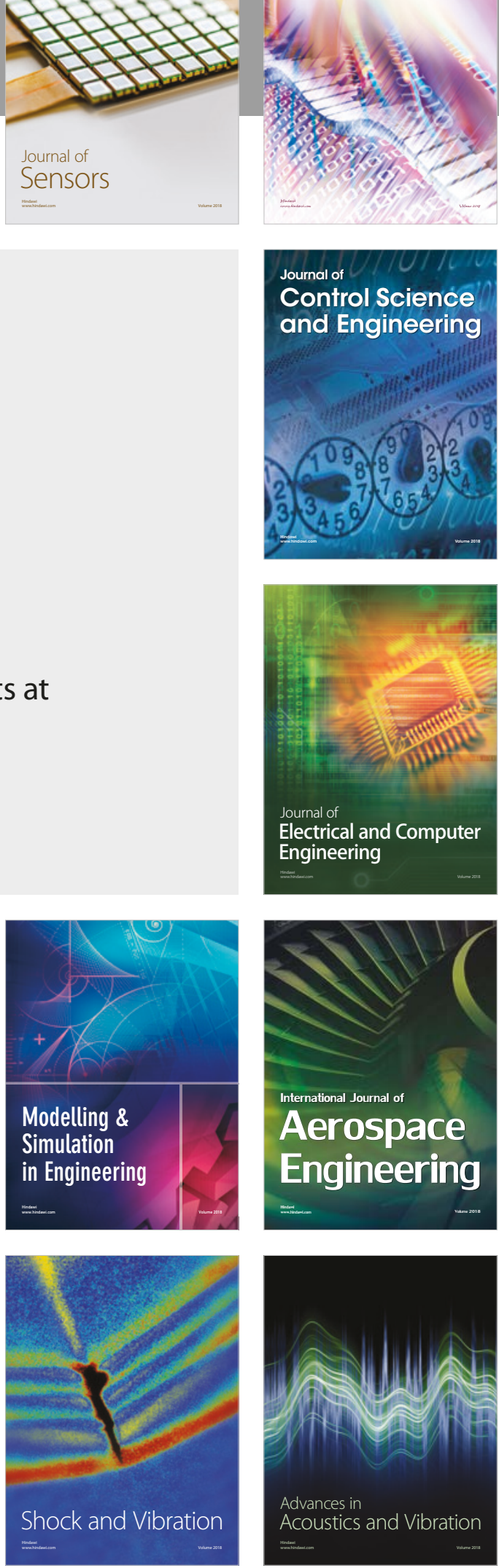